\title{
TI.32.1
}

\section{LDAP Representations of eduCourse Attributes and an Auxiliary Object Class}

- PDF: internet2-mace-courseid-educourse-ldap-200505.pdf

- HTML: internet2-mace-courseid-educourse-ldap-200505.html

\section{More Information}

\begin{tabular}{|l|l|}
\hline Repository ID & TI.32.1 \\
\hline Persistent URL & http://doi.org/10.26869/TI.32.1 \\
\hline Title & LDAP Representations of eduCourse Attributes and an Auxiliary Object Class \\
\hline Authors & Keith Hazelton \\
\hline Sponsor & MACE \\
\hline Review & \\
\hline Status & Legacy \\
\hline Publish Date & $5 / 17 / 2005$ \\
\hline DOI & $10.26869 /$ TI.32.1 \\
\hline Signature & \\
\hline Deprecated & No \\
\hline Future Review & \\
\hline Supersedes & \\
\hline Format & PDF \\
\hline Related Docs & \\
\hline Development Location & \\
\hline IP Framework & \\
\hline Subject Tags & \\
\hline Notes & \\
\hline
\end{tabular}

